Cinémas

Revue d'études cinématographiques

Journal of Film Studies

\title{
Considérations sur la portée éthique des propositions de Christian Metz pour une énonciation impersonnelle au cinéma
}

\section{Alain Lacasse}

Volume 4, numéro 3, printemps 1994

Questions sur l'éthique au cinéma

URI : https://id.erudit.org/iderudit/1001039ar

DOI : https://doi.org/10.7202/1001039ar

Aller au sommaire du numéro

Éditeur(s)

Cinémas

ISSN

1181-6945 (imprimé)

1705-6500 (numérique)

Découvrir la revue

Citer cet article

Lacasse, A. (1994). Considérations sur la portée éthique des propositions de Christian Metz pour une énonciation impersonnelle au cinéma. Cinémas, 4(3), 85-97. https://doi.org/10.7202/1001039ar
Résumé de l'article

Cet article, à partir d'une étude des implications éthiques de la nouvelle définition de l'énonciation que propose Metz dans son ouvrage L'Énonciation impersonnelle ou le site du film, porte sur l'examen des conséquences du glissement de l'importance conférée traditionnellement, dans le langage, aux marques de subjectivité vers les manifestations de la réflexivité du langage, et cherche à évaluer la contribution éthique respective de ces deux grandes catégories de traces de fabrication du film dans le film. 


\section{Considérations sur la portée éthique des propositions de Christian Metz pour une énonciation impersonnelle au cinéma}

\section{Alain Lacasse}

\section{RÉSUMÉ}

Cet article, à partir d'une étude des implications éthiques de la nouvelle définition de l'énonciation que propose Metz dans son ouvrage L'Énonciation impersonnelle ou le site $d u$ film, porte sur l'examen des conséquences du glissement de l'importance conférée traditionnellement, dans le langage, aux marques de subjectivité vers les manifestations de la réflexivité du langage, et cherche à évaluer la contribution éthique respective de ces deux grandes catégories de traces de fabrication du film dans le film.

\section{ABSTRACT}

This article, based on a study of the ethical implications of the new definition of enunciation that Metz proposes in his latest work, L'Énonciation impersonnelle ou le site $d u$ film, examines the consequences of the slippage away from the importance traditionally accorded to indications of subjectivity in language in favour of manifestations of the reflexivity of language. It seeks to evaluate the respective ethical contributions of these two broad categories of traces of the making of a film incorporated in the film. 
La problématique de l'énonciation occupe une place importante au sein des études cinématographiques des 20 dernières années. On la retrouve d'ailleurs au cœur de la controverse opposant les partisans d'une étude du cinéma en tant que langage aux autres, qui prônent davantage l'étude du cinéma en tant que "mode de représentation", cherchant là à débarrasser la théorie du cinéma des modèles anthropomorphiques empruntés à la linguistique ou aux études littéraires. Le nom de l'Américain David Bordwell restera longtemps associé de façon étroite au second groupe, celui-ci n'ayant pas manqué d'alimenter la polémique autour de cette question. Mais comme le souligne Christian Metz dans son dernier ouvrage, L'Énonciation impersonnelle ou le site du film, Bordwell n'est pas le seul à suggérer depuis longtemps que les études cinématographiques se "distancient" des études littéraires et de leurs modèles. Metz souligne en l'occurrence la position de Gérard Genette, théoricien émérite de la littérature, qui n’a jamais voulu considérer le film en tant que récit, car il n'est pas un être de langue (p. 19).

Avec L'Énonciation impersonnelle ou le site du film, Metz a voulu faire le point sur la question de l'énonciation au cinéma et par la proposition à laquelle il parvient, l'éminent théoricien du langage cinématographique nous apparaît faire un grand pas en direction des thèses de Bordwell. En effet, bien que dans les premières pages de son livre, il écrive que l'énonciation consiste en un acte sémiologique par lequel certaines parties d'un texte nous parlent de ce texte comme d'un acte (p. 21), Metz ne revient pas à cette définition à la fin de son ouvrage. Est-ce tout simplement parce qu'il n'a pas vu la nécessité de l'intégrer aux propos alors tenus? Quoi qu'il en soit, il nous apparaît que s'il avait dû ou avait voulu le faire, il lui aurait certes fallu remplacer le mot acte par les mots processus ou fonctionnement (p. 199) sur lesquels il se fixe finalement. Car comme l'indique le titre de son ouvrage, c'est sur une définition dépersonnifiée, dépersonnalisée de l'énonciation qu'il s'arrête en fin de parcours. Il dissocie pour de bon les concepts d'énonciateur et d'énonciataire de celui d'énonciation et il libère ainsi le fonctionnement de l'énonciation des instances qui y étaient rattachées puisque, soutient-il, l'énonciateur n'est pas un émetteur (une personne physique) et l'énonciataire n'est pas un 
récepteur. Il n'y a entre eux aucune interrelation réelle possible. Le concept d'énonciation rompt ainsi pour de bon avec celui de communication et l'objectif de Metz semble être de le libérer définitivement de tout caractère anthropomorphique. L'énonciation consiste en une fonction, elle réside dans le fait d'énoncer (p. 187). Dans le film, pour Metz, l'énonciation consiste d'abord en un premier niveau de discours qui est toujours impersonnel, en quelque sorte désincarné (p. 209).

Les propositions de Metz sur l'énonciation sont d'importance et il nous est apparu intéressant de tenter d'évaluer les implications éthiques du modèle qu'il nous propose, car celui-ci suggère en l'occurrence d'éliminer toute notion d'instance de production du discours filmique. Si l'on considère de près le fonctionnement de ce type de discours, toute idée d'une personne qui en serait à l'origine disparaît au profit d'un certain processus discursif autonome. Il n'est même plus question d'instances abstraites. Lénonciation fonctionne indépendamment de toute source identifiable ou le moindrement manifeste. Elle consiste en une fonction, terme dont nous serions porté à réduire le sens à une seule des définitions que nous en propose le Robert, "action propre d'un instrument, d'une machine", au détriment de la définition à caractère linguistique que le dictionnaire nous offre également. Or, c'est précisément cette définition impersonnelle, instrumentale, "machinique" de l'énonciation cinématographique qui suscite le questionnement éthique. D'autant plus qu'en dissociant le concept d'énonciation filmique de la notion de sujet, l'auteur propose de le soulager du même coup de tout rapport à la "deixis", notion traditionnellement associée aux marques de subjectivité dans le langage. Selon lui, on ne retrouve pas de traces d'une véritable deixis dans le texte du film. La deixis de l'énonciation " [...] est simulée, ses marques principales sont des replis du texte sur lui-même» (p. 200). En conséquence, il propose de déplacer l'accent mis depuis longtemps sur la deixis vers la réflexivité, en l'espèce la réflexion de l'énonciation au sein de l'énoncé, " [...] sans laquelle une phrase à déictiques n'est même pas interprétable» (p. 209), précise-t-il.

C'est ce glissement de l'importance que l'on confere habituellement dans le langage aux marques de subjectivité vers les 
manifestations de la réflexivité que nous désirons considérer ici de plus près. De fait, nous tenterons d'évaluer la contribution éthique respective de ces deux grandes catégories de traces de fabrication du film dans le film, pour ensuite évaluer ce que l'importance plus grande reconnue par Metz à la seconde dans le texte du film implique pour l'étude sur la portée éthique du langage cinématographique en général.

Rappelons que ce genre de considérations et leur rapport à la notion d'énonciation ont souvent été abordés par le passé sous le thème de la transparence. Ce dernier thème de la transparence a été particulièrement cher au discours sur le film des années soixante-dix, un discours qui faisait souvent office de critique sociale, politique et morale. À ce sujet, nous pourrions dire de la réflexion de Metz qu'elle entraîne un peu les même effets sur cette notion de transparence que la réflexion de David Bordwell sur le même sujet et dont Metz dit qu'elle la " [...] conforte par des voies en parties neuves, [...]" (p. 179). Il cite Bordwell qui soutient, dans Narration in the Fiction Film, que les films hollywoodiens les plus "transparents" portent encore des marques visibles de narration. Metz est tout à fait d'accord avec lui et c'est en l'occurrence pour tenir compte de considérations de ce type qu'il juge préférable de ne pas restreindre la définition de l'énonciation à la seule question de la subjectivité dans le langage et de l'étendre au concept plus "objectif" de réflexivité. Selon son point de vue, il faut cesser d'associer le concept d'énonciation au seul discours filmique parsemé de traces de subjectivité pour le mettre, ensuite, en opposition "quasi définitive" avec le film "transparent». La transparence du film ou de l'énonciation dans le film se joue à plusieurs niveaux et la définition de ce concept fait davantage référence à des questions de réflexivité qu'à des notions de subjectivité dans le langage.

Au début de L'Énonciation impersonnelle ou le site du film, Metz dit de l'énonciation qu'elle consiste en un " [...] acte sémiologique par lequel certaines parties d'un texte nous parlent de ce texte comme d'un acte» (p. 21). Aussi nous est-il apparu tout à fait pertinent de chercher quelque écho à cette définition dans la théorie des actes de langage issue de recherches en philosophie analytique. D'autant plus que Metz lui-même, dans 
son ouvrage, nous y encourageait déjà en faisant référence à la philosophie analytique à quelques reprises (p. 189, 191, 209 et 210). Deux textes tirés d'une publication sur le sujet intitulée Théorie des actes de langages. Éthique et droit nous ont guidé sur cette voie. Il s'agit de "L'analyse des énoncés moraux avant Austin" de Francis Jacques et de "La pensée d'Austin et son originalité par rapport à la philosophie analytique antérieure " de François Recanati.

Il faut préciser immédiatement avec Francis Jacques que la théorie des actes de langage et, partant, la philosophie analytique ne doivent être confondues " [...] ni avec la linguistique - qui ferait du langage un objet - ni avec la philosophie du langage - qui traite le langage comme un médium en analysant le rapport tout à fait général entre les mots et les choses" (p. 62). La philosophie analytique est un produit de la réflexion éthique anglo-saxonne qui, au lieu de s'arrêter à l'étude de la nature de l'expérience morale, de l'origine des normes ou du changement des valeurs, s'est davantage concentrée sur l'analyse du langage moral (p. 55), c'est-à-dire des structures linguistiques qui portent cette expérience morale. On appela vite méta-éthiques les recherches qui s'efforcent de rendre compte du discours moral dans ses aspects sémantiques (p. 61). Cette démarche fait écho, d'une certaine façon, à la nôtre puisque nous cherchons particulièrement à vérifier en quoi les structures d'énonciation du film participent de la valeur éthique de celui-ci. Les textes de Recanati et de Jacques qui rendent compte du cheminement effectué par la philosophie analytique pendant une certaine période témoignent à notre avis de la valeur éthique qu'il est possible d'associer respectivement à la deixis et à la réflexivité du langage.

Le texte sur lequel est principalement basé notre étude est celui de François Recanati, qui nous introduit aux travaux de John Langshaw Austin et qui tente, comme l'indique son titre, de dégager l'originalité de la pensée d'Austin par rapport à la philosophie analytique antérieure. Nous avons retenu des propos de Recanati ceux qui nous sont apparus particulièrement éclairants sur la façon dont Austin a abordé la question de la réflexivité dans le langage après que ses prédécesseurs aient pour leur part réfléchi sur la deixis. La mise en rapport de ces 
problématiques avec les préoccupations éthiques de base de la philosophie analytique nous fournissent, croyons-nous, des indications pertinentes sur la contribution réelle que nous devrions reconnaître à la valeur éthique d'un texte, qu'il soit littéraire ou cinématographique.

Recanati rappelle d'abord les origines communes de la logique mathématique et de la philosophie analytique. Il mentionne que les mathématiciens-philosophes Gottlob Frege et Bertrand Russell, pionniers de la logique mathématique, furent également les fondateurs de la philosophie analytique et il précise que la logique joue encore, de nos jours, un rôle central dans la philosophie analytique (p. 19). Il nous informe ensuite du fait que c'est à la logique mathématique que ces pionniers de la philosophie analytique ont emprunté leur préoccupation éthique de base du langage, pour le vrai ou pour le faux.

De fait, les penseurs de la première analyse, c'est-à-dire de la philosophie analytique en rigueur jusqu'à la Seconde Guerre mondiale, concevaient tout système logique comme un langage, avec son vocabulaire et ses règles syntaxiques de bonne formation pour les expressions complexes et les propositions (p. 20). L'idée était que tous ces langages artificiels de la logique combinés aux langues naturelles consistaient en des manifestations d'un langage unique, "le" langage.

Tant que ces représentants de la première analyse s'en sont tenus à l'étude des langages logiques, le modèle tenait bien. C'est lorsqu'on a tenté de l'appliquer réellement aux langues naturelles que l'entreprise s'est butée à de sérieuses difficultés. Car dans le langage logique mathématique proposé par Russell, par exemple, tous les énoncés sont des affirmations. Il n'existe pas d'énoncés impératifs ou interrogatifs. Les phrases du langage de la logique sont ainsi toutes vraies et celles-ci lui conferent en conséquence un statut éthique remarquable. Les énoncés du langage logique ne communiquent que des vérités. Impossible de mentir en utilisant un tel langage. Pas surprenant que ses concepteurs aient cru pouvoir l'associer à ce qu'ils considéraient être "le " langage.

Les difficultés sont apparues lorsque les logiciens se sont vus confrontés de plus en plus, dans leurs recherches, à la diversité 
des systèmes logiques et, du coup, à la pluralité des langages qui en découle. Ils s'étaient déjà interrogés auparavant sur les propriétés du langage ordinaire, des langues naturelles, sur leur caractère obscur et compliqué, leurs apparences trompeuses et leur structure qui n'est pas évidente, qualités qui ne peuvent que peser très lourd lors de leur évaluation dans une perspective éthique. Ils sont de fait arrivés à la conclusion que le langage ordinaire ne se distingue des langages artificiels de la logique que par ses défauts (p. 20). De fait, on considérait que le langage ordinaire souffrait d'un défaut tout à fait majeur en proposant des phrases qui ne sont pas ou vraies ou fausses ni clairement identifiables comme telles. "Pour les philosophes de l'ancienne analyse, les phrases représentent des états de choses et sont vraies ou fausses selon que ces états de choses sont réels ou ne le sont pas" (p. 21).

Cette problématique de transmission de la vérité par le langage s'échelonnera sur un certain nombre d'étapes et passera par toutes sortes de modifications. Recanati en fait l'énumération et rend compte du processus dans toute sa complexité. Il serait inutile de reprendre ici sa démonstration dans le détail. Nous reviendrons néanmoins à quelques-unes de ces grandes étapes du développement de la philosophie analytique depuis Frege et Russell jusqu'à Austin dans la mesure où celles-ci nous éclairent précisément sur la portée éthique des marques de réflexivité ou de subjectivité dans le langage.

Les philosophes du langage ordinaire feront vite remarquer à ceux de l'ancienne analyse que dans les langues naturelles, les phrases ne se situent pas d'un bloc du côté du vrai ou du côté du faux. Impossible, puisqu'il faut entre autres distinguer la phrase (qui a un sens) de l'énoncé de cette phrase qui rend compte de son emploi discursif. Considérant que c'est l'énoncé qui représente un état de choses, on croira d'abord que c'est seulement lui qui est vrai ou faux selon le contexte d'énonciation, sans jamais être automatiquement l'un ou l'autre. Bien entendu, on déterminera ultérieurement que l'énoncé ne détient pas à lui seul le privilège de semer le doute sur le caractère vrai ou faux de la phrase, la phrase elle-même pouvant témoigner de non-sens. Cependant, nous désirons faire porter notre attention 
ici sur la pensée des philosophes de l'ancienne analyse pour qui c'est avec l'énoncé qu'intervient la problématique des marques de subjectivité dans le langage sous la catégorie des éléments "indexicaux». Il est dit que "l'indexicalité est l'une des choses qui distinguent les langues naturelles du langage des Principia Mathematica " (p. 21), en obligeant en l'occurrence à distinguer la phrase de l'énoncé. Ceci revient à dire que les marques de subjectivité dans le langage sont parmi les éléments qui, au lieu de contribuer à faire en sorte que la phrase soit de toute évidence vraie ou fausse, lui conferent une certaine ambiguïté et jettent le doute sur les vérités qu'elles véhiculent. Recanati précise: l'«indexicalité" constitue l'un des principaux facteurs "[...] qui, dans les langues naturelles, empêche[nt] d'identifier la phrase au sens grammatical et la phrase au sens logique, c'està-dire l'affirmation vraie ou fausse" (p. 23). À cause des éléments indexicaux qu'il implique, on dira ainsi de l'énoncé qu'il «[...] ne véhicule aucun sens cognitif, le sens cognitif étant défini par le fait que seules les affirmations vraies ou fausses en ont un" (p. 24). Les marques de subjectivité contribuent ainsi de façon importante à ce que la syntaxe des langues naturelles ne soit pas une "syntaxe logique".

Ainsi, dans l'histoire de la philosophie analytique, du moins selon la façon dont Recanati en rend compte, l'indexicalité (ou la deixis) a été reconnue comme l'un des facteurs premiers qui, d'un point de vue éthique, font que le langage ordinaire entretient sans cesse le doute sur le vrai ou faux. Nous nous en tiendrons pour le moment à cette constatation d'ordre général sur les implications éthiques de base associées à la deixis ou aux marques de subjectivité dans le langage pour ensuite examiner la façon dont la même discipline a fait intervenir la question de réflexivité du langage en regard de la même problématique.

La nécessité devenue évidente de dissocier la phrase de l'énoncé et l'impossibilité qui est apparue ensuite d'associer clairement l'une ou l'autre au seul vrai ou au seul faux allaient inciter par la suite les philosophes à dissocier les aspects cognitif et pragmatique (ou non sémantique) du langage. Reconnaître un sens cognitif à une phrase consistait à reconnaître son caractère vrai ou faux. La fonction pragmatique d'une autre se 
reconnaissait à l'inverse à son caractère vrai ou faux incertain. C'est à ce moment, selon Recanati, que s'est effectuée la véritable transition du positivisme logique à la philosophie du langage ordinaire et qu'est intervenu Austin avec son concept d'énoncé "performatif".

L'énoncé performatif, selon Austin, est un énoncé essentiellement vrai qui tient sa vérité non plus de sa participation à un système logique, mais de sa fonction sociale institutionnelle. Les performatifs servent à accomplir des actes sociaux et institutionnels tels se marier ou condamner quelqu'un à la prison. C'est la sanction sociale qui concède sa vérité au performatif et à l'acte de langage qu'il constitue. On voit bien ici ce que Recanati entend par la véritable transition du positivisme logique à la philosophie du langage ordinaire qu'implique le concept de performatif. Or, ce vers quoi s'orientera plus tard la théorie des actes de langage avec Austin, c'est, selon lui, vers la constatation générale à l'effet qu'on "[...] ne peut accomplir légitimement n'importe quel acte de parole dans n'importe quel contexte $[\ldots]$ " (p. 34). Les actes de parole, au même titre que les actes institutionnels, seront alors eux aussi reconnus comme pouvant se prêter à une sanction sociale. Ils pourront trouver là, à leur tour, leur félicité, leur vérité.

Nous n'irons pas plus loin dans ce genre de considérations sur l'intersection entre le langage et l'institution sociale. Notre intérêt porte plus spécifiquement sur les marques de subjectivité et de réflexivité dans le langage et il n'est pas de notre intention d'aller plus en profondeur dans l'analyse de leur rôle éthique en regard plus spécifiquement de la fonction sociale du discours qui les porte. Revenons pour l'instant sur l'intersection du logique et du social comme modes de sanction de la vérité dans le langage et sur l'énoncé "performatif" d'Austin qui y correspond et avec lequel la philosophie analytique franchit une étape importante. Du point de vue éthique, ce qui rend le performatif intéressant pour Austin, c'est que celui-ci fait partie de la catégorie des énoncés non cognitifs, ceux qu'on ne peut ramener automatiquement ni à du vrai ni à du faux. À ce titre, c'est à sa fonction sociale qu'il doit sa vérité. La philosophie analytique s'engage alors avec lui vers un glissement, dans ses critères de 
vérité, du logique au pragmatique et à l'empirisme. Néanmoins, Austin semble entretenir pour un temps, si on lit en diagonale les propos de Recanati, une certaine nostalgie des définitions logiques du vrai et du faux puisqu'il privilégie d'abord, parmi les énoncés performatifs, ceux qui sont réflexifs. C'est là qu'il fait intervenir le concept de réflexivité, à cheval sur les fonctions logiques et sociales du langage. De fait, c'est comme si, bien qu'affirmant que la vérité du performatif tient principalement à son rapport au social, Austin voulait tout de même lui reconnaître une certaine vérité de type logique. Il précise: "Pour qu'on ait une description ou vraie ou fausse, il faut qu'on ait deux choses, l'état de choses représenté et l'énoncé qui le représente. Mais un énoncé performatif constitue l'état de choses dont il parle, et ne s'en distingue donc pas" (Recanati, p. 27). Lorsque quelqu'un, par exemple, dit: "Je m'excuse", il accomplit à la fois l'acte de s'excuser et il énonce ce qu'il est en train de faire. En ceci consiste le caractère réflexif du performatif qui le rend spécifique et participe de sa vérité.

Austin réalisera bientôt que tout n'est pas si simple et que lorsqu'il est question d'acte, par exemple, l'évaluation en termes de fausseté et de vérité qui s'applique davantage aux énoncés cognitifs doit être remplacée par une évaluation en termes de succès : en tant qu'acte, un énoncé performatif doit être reconnu comme appartenant plutôt à la catégorie des pragmatiques et doit être évalué en termes de réussite (réussi ou raté) plutôt que de vérité (vrai ou faux) (p. 27). Il jugera plus tard que les fonctions cognitives et pragmatiques ne se rapportent pas nécessairement à deux catégories d'énoncés, mais peuvent s'appliquer à un seul et même énoncé. Comme il reconnaîtra que la réflexivité ne concerne pas que les actes institutionnels, ces actes sociaux réglés par des conventions auxquels il l'associait à l'origine. Il conviendra finalement qu'elle puisse s'appliquer aux simples actes de parole.

L’objectif premier de cette étude, rappelons-le, visait à poser le problème de la contribution de l'énonciation à la portée éthique d'un texte. Examinée du point de vue de ses champs de manifestation principaux, ceux des marques de subjectivité et des traces de réflexivité, il s'agissait pour nous de démontrer en 
quoi la philosophie analytique ou plus précisément le résumé de son évolution que nous propose, en l'occurrence, le texte de Recanati nous suggère certaines voies de recherche pertinentes. À ce sujet, nous tirerons provisoirement les conclusions suivantes.

Il nous apparaît qu'à des moments importants de l'histoire de la philosophie analytique, autant la problématique des marques de subjectivité dans le langage que celle de la réflexivité ont été mises en rapport avec le questionnement éthique sur la vérité dans le langage. Les questions de deixis et de réflexivité ont en quelque sorte constitué deux volets d'un même questionnement sur la vérité transmise par le langage. Ce questionnement a finalement abouti à l'échec d'une tentative de définition logique du vrai dans le langage, échec auquel contribuent à la fois la deixis et la réflexivité, mais chacune à sa façon. Et voilà, nous retiendrons surtout la différence de leur contribution à ce niveau pour la mettre en rapport avec les nouveaux critères de vérité que la philosophie analytique a ensuite appliqués au langage.

En présentant, tel que nous le propose Austin, l'acte performatif dans son rapport à la réflexivité, nous avons insisté sur le fait que la philosophie analytique arrivait là à l'intersection du logique et du social comme modes de sanction de la vérité dans le langage. De fait, on aurait pu tenir un peu les mêmes propos lorsqu'il a été question de la deixis puisqu'en plus de dire de celle-ci qu'elle empêchait que la phrase soit de toute évidence vraie ou fausse, qu'elle lui conférait une certaine ambiguïté, nous avons précisé que la deixis renvoyait au contexte d'énonciation. Indirectement, le mot contexte aurait pu nous conduire à des considérations d'ordre social. La deixis comme la réflexivité se révèlent ainsi toutes deux pertinentes à considérer en regard de ce qu'Austin aurait appelé la fonction pragmatique du langage.

Il n'en est toutefois pas de même dans la mise en rapport de chacune d'elles à la fonction du langage que nous pourrions dire cognitive, pour reprendre Austin, et qui a trait à une définition logique du vrai. À ce sujet, nous avons dit que, pour le performatif, son caractère réflexif ne pouvait pas, en définitive, être 
garant de la vérité puisque le performatif consistant en un acte, cet acte ne peut être vrai ou faux, mais il doit plutôt avoir lieu ou non. Ce qui revient finalement, si l'on s'en tient à la stricte définition d'un performatif phrasique, à éloigner la problématique de la réflexivité de celle de la vérité.

Alors qu'il n'en est pas ainsi en ce qui concerne les marques de subjectivité dans le langage ou la deixis. Car lorsque l'on affirme que, pour les prédécesseurs d'Austin, la deixis fait en sorte que la phrase ne soit pas automatiquement vraie ou fausse, qu'elle y sème l'ambiguïté à ce niveau, nous n'éliminons pas du même coup son rapport à la problématique du vrai et du faux. Elle y demeure associée d'une façon qu'il faut continuer à évaluer et dont il faudrait chercher les traces dans l'histoire plus récente de la philosophie analytique.

Ces constatations sur les rapports différents de la deixis et de la réflexivité aux considérations éthiques de vérité du langage nous incitent à l'expression d'un commentaire qui nous apparaît particulièrement pertinent à l'étude du langage filmique, surtout si l'on tient compte de la spécificité de l'énonciation cinématographique que Christian Metz propose dans L'Énonciation impersonnelle ou le site du film. En effet, si l'énonciation filmique se manifeste essentiellement dans le texte par sa réflexivité plutôt que par des marques de subjectivité, comme Metz le soutient, nous pourrions en déduire que l'énonciation filmique porte moins en elle-même la marque de préoccupations éthiques que ne le peut l'énonciation en littérature, par exemple, où la deixis est constamment manifeste.

Une telle observation, si elle est valable, ne serait pas sans conséquence dans une étude plus approfondie des implications éthiques internes au langage cinématographique en regard de considérations plus pragmatiques, à caractère social. Pour ne citer qu'un exemple, le texte de Francis Jacques "Lanalyse des énoncés moraux avant Austin" tente précisément de mettre en rapport certains aspects sémantiques (cognitifs) du langage et leur utilisation en contexte. Or, Jacques conclut que pour constituer un véritable "discours moral justifié", il faudrait recourir à un aspect de la deixis trop peu considéré jusqu'à maintenant dans ce genre de projet: le registre pronominal. Il faudrait faire 
appel " [...] aux ressources supplémentaires offertes à l'analyse par une pragmatique de l'interlocution" (p. 87). Que penser alors des prédispositions du cinéma à produire ce type de "discours moral justifié" si, comme le propose Metz, il consiste en un langage qui ne dispose pas véritablement des ressources de la deixis et encore moins d'un véritable registre pronominal puisque ce qui caractérise son énonciation, c'est son caractère impersonnel, "dépersonnifié ", dépersonnalisé.

\section{Université Laval}

\section{OUVRAGES CITÉS}

Bordwell, David. Narration in the Fiction Film. Madison: The University of Winconsin Press, 1985, 370 p.

Jacques, Francis. "L'analyse des énoncés moraux avant Austin", dans Paul Amselek (direction), Théorie des actes de langage. Éthique et droit. Paris: P.U.F., 1986.

Metz, Christian. L'Énonciation impersonnelle ou le site du film. Paris: Méridiens Klincksieck, 1991, 228 p.

Recanati, François. "La pensée d'Austin et son originalité par rapport à la philosophie analytique antérieure", dans Paul Amselek (direction), Théorie des actes de langage. Ethique et droit. Paris: P.U.F., 1986. 